\title{
Construcción, transformación y reproducción de paisajes campesinos. Aportes desde múltiples escalas
}

Enrique Moreno*

\section{Resumen}

El uso de los Sistemas de Información Geográfica (SIG) y otras herramientas analíticas para el estudio del paisaje ha tenido un fuerte auge en los últimos años, permitiendo caracterizar las distintas maneras en que las poblaciones humanas se relacionaron con el entorno y sus características. Pero a la vez existen algunas prácticas sociales, tales como el pastoreo y la agricultura, que tienden a determinar el uso del espacio por parte de las poblaciones humanas, siendo dejadas de lado algunas otras prácticas sociales que constituyen relaciones de largo término entre las personas y el paisaje y que son relevantes para la reproducción social cotidiana. En este trabajo pretendemos reflexionar en torno a la manera en que podemos visualizar, estudiar y preguntarnos acerca de múltiples prácticas sociales, tales como la cacería, la recolección de leñas, la obtención de materias primas y su interacción con el ambiente y las relaciones que allí se establecen. Para ello nos centraremos en el análisis realizado en la quebrada de Antofalla (departamento Antofagasta de la Sierra, Catamarca) para evaluar estas diversas prácticas y su profundidad histórica, y así evaluar múltiples escalas de análisis para su estudio.

\section{Construction, transformation, and reproduction of peasant landscapes: a multi-scale contribution}

Recibido: 13 de agosto de 2017

Aceptado:

19 de febrero de 2018

\section{Palabras clave}

\section{Paisaje}

Prácticas sociales

Sistemas de Información Geográfica

Campesinos

\begin{abstract}
The use of Geographical Information Systems (GIS) and other analytical tools for the study of landscapes has been on the increase over the last years. GIS allows us to characterize the different ways by which human populations related to the environment and the characteristics therein. Concomitantly, there are certain social practices, such as herding and agriculture, which tend to determine the use of the space by human populations, relegating other social practices that also constitute long-term relations between people and landscapes, which are also important for daily social reproduction.
\end{abstract}

\section{Keywords}

Landscape Social practices Geographic Information Systems Peasants

* Centro de Investigaciones y Transferencia (CITCA), Universidad Nacional de Catamarca (UNCa) - CONICET / Escuela de Arqueología, Universidad Nacional de Catamarca (UNCa). Prado 366 (CP K470oBDH). San Fernando del Valle de Catamarca, Catamarca, Argentina. E-mail: enalmor@gmail.com 
1. En nuestro país se desarrollaron varios simposios y encuentros destinados al estudio del paisaje en Congresos Nacionales, Internacionales y Reuniones locales, así como también publicaciones específicas que tratan el tema.
In this article, we reflect and assess the means by which we can visualize, study and question the other multiple social practices that existed in these societies such as hunting, hardwood and lithic raw material procurement, and its interaction with the environment and the relationships thus established. In doing so, we present our research from the Antofalla Valley (Department of Antofagasta de la Sierra, Catamarca). Here we visualize these multiple practices, their historical depth, and assess the multiple scales of analysis needed for their study.

\section{Introducción}

Desde hace varios años el estudio del paisaje en arqueología se ha convertido en un eje central de múltiples investigaciones, discutido desde diversas perspectivas teóricas y metodológicas, e incluso ha servido como foco para la realización de eventos científicos, mesas redondas y simposios ${ }^{1}$ (Aldenderfer y Maschner, 1996; Anschuetz, Wilshusen y Scheick, 2001; Baena Preysler, Blasco Bosqued y Quesada Saenz, 1997; Bruno y Thomas, 2008; Criado Boado, 1999; Curtoni, 2007; Figuerero Torres e Izeta, 2013; García Sanjuán, 2005; Gillings, 2012; Ingold, 1993; Kvamme, 1999; Llobera, 2012; Maschner, 1996; Pastor, Murrieta Flores y García Sanjuán, 2013; Ucko y Leyton, 1999). De las múltiples vías de comprensión del paisaje y de la multiplicidad de herramientas utilizadas para su análisis surgió la idea del presente artículo, que también se nutre de las preguntas y del propio recorrido que siguió la investigación en el caso de estudio que desarrollaremos a continuación. Este trabajo persigue el objetivo de pensar la manera en que los paisajes son experimentados, construidos y vividos en articulación con las prácticas sociales y en la interacción entre las poblaciones humanas y los múltiples seres que lo habitan. En particular, parte de una reflexión en torno de la manera en que algunos paisajes son definidos desde la arqueología y desde las múltiples herramientas analíticas para analizarlo. Cuando nos ubicamos en ambientes puneños, particularmente, resalta su caracterización como un espacio agreste, desierto, con pocas posibilidades para la reproducción social, y donde las estrategias de producción suponen una preeminencia de algunas prácticas, particularmente el pastoreo o la agricultura. En este sentido, estas prácticas son las que habrían definido la construcción y reproducción del paisaje cultural, definiendo pautas de asentamiento, áreas de explotación de recursos, estructuras arquitectónicas, etc. Sin embargo, resulta claro que además de estas prácticas, imprescindibles para la reproducción social, existen otras importantes actividades que demarcan, construyen o modifican el paisaje, y por lo tanto, pueden ser observadas mediante técnicas analíticas específicas. Para ello, creemos que el concepto de paisaje campesino puede servir de marco referencial a la hora de definir estas interacciones entre las poblaciones humanas y el entorno. Por lo tanto, nuestro objetivo aquí radica en definir de qué manera el paisaje es construido, vivido y experimentado en el marco de diversas prácticas sociales que dan sentido a la reproducción doméstica.

Para alcanzar este objetivo nos centraremos en un caso de estudio que desarrollamos hace algunos años y en dónde surgieron algunas de estas reflexiones mediando entre las múltiples prácticas sociales que podíamos observar e interpretar y la forma en que las herramientas analíticas del estudio del paisaje nos permitían caracterizar y divulgar. El caso de estudio es el de la quebrada de Antofalla, donde fueron definidas claras evidencias de agricultura y pastoreo, pero donde también la cacería, la recolección de leña, la obtención de materias primas líticas para la manufactura de instrumentos y otras prácticas eran importantes demarcadores del paisaje y de la vida cotidiana. 


\section{El paisaje arqueológico. Implicancias teóricas y metodológicas}

Son abundantes los trabajos que han planteado la importancia del concepto de paisaje en la arqueología, tanto a nivel local como a escala global (Aldenderfer y Maschner, 1996; Anschuetz et al., 2001; Baena Preysler et al., 1997; Bruno y Thomas, 2008; Criado Boado, 1999; Curtoni, 2007; Figuerero Torres e Izeta, 2013; García Sanjuán, 2005; Ingold, 1993; Kvamme, 1999; Maschner, 1996; Pastor et al., 2013; Ucko y Leyton, 1999), por lo que aquí no nos centraremos en ello, sino más bien comenzaremos nuestro camino planteando los cambios que han sido propuestos en las dinámicas sociales y, por lo tanto, en el paisaje a partir de la domesticación de animales y plantas (Diamond, 2002; Harris, 1996; Korstanje y Yacobaccio, 2007; Korstanje, Quesada, Franco Salvi, Lema y Maloberti, 2015; Lema, 2008, 2014; Mengoni Goñalons y Yacobaccio, 2006; Olivera, 1991; Yacobaccio y Vilá, 2002). Sin embargo, es necesario realizar una aclaración con respecto a las características de los paisajes analizados aquí: debido a las características del caso propuesto, nos centraremos en el paisaje de la Puna seca catamarqueña. Aunque es posible desarrollar las reflexiones que aquí se vierten para otros paisajes con otras dinámicas ambientales y trayectorias históricas.

Una de las principales características de la adopción de la agricultura y el pastoreo en el área puneña ha sido la marcada sedentarización de las poblaciones, así como también el establecimiento de estructuras arquitectónicas y productivas, tendientes al desarrollo de dichas actividades. Corrales, redes de riego, canchones de cultivo, melgas, puestos, por nombrar sólo algunos, son claros ejemplos de una transformación del paisaje social, con demarcaciones materiales permanentes y duraderas, que definen claramente su funcionalidad. Esto ha generado diversos modelos de ocupación del territorio y ha abonado la idea de la construcción de un paisaje definido en términos de la actividad agrícola y/o pastoril (ver Di Lullo, 2010; Franco Salvi y Berberian, 2011; Göbel, 2002; Korstanje et al., 2015; Lema, 2013; Olivera, 1991; Quesada, 2007, 2010; Yacobaccio, Madero, Malmierca y Reigadas, 1997-1998; Yacobaccio et al., 2011).

Pero también se ha demarcado la manera en que se vive, transforma y articula el espacio socio-productivo en estos ambientes. Por ejemplo, Olivera (1991) planteó el modelo de sedentarismo dinámico para la región de Antofagasta de la Sierra, vinculado con un uso del espacio en el cual los diferentes pisos altitudinales se articulaban en una lógica de producción pastoril, donde los fondos de la cuenca eran utilizados para la instalación humana durante mayor cantidad de tiempo y donde también se realizaban prácticas agrícolas, mientras que las quebradas de altura se aprovechaban para la alimentación de los rebaños en ciertas épocas del año y para la cacería de animales silvestres. La idea de articulación de espacios productivos aprovechando las características del paisaje es compartida por varias investigaciones, en donde se plantea la presencia de espacios agrícolas, generalmente ubicados en fondos de cuencas, con suelos aptos y aporte hídrico, imbricada con la ubicación de puestos en altura hacia donde son trasladados los animales para que pasten en ciertas épocas del año (Cladera, 2013; Delfino, 2001; Göbel, 2002; Tomasi, 2011; Yacobaccio et al., 1997-1998, 2011). Estos autores plantean la vinculación, más allá de ciertas diferencias en la denominación de las construcciones, entre bases residenciales, vinculadas con los espacios agrícolas y con la reproducción doméstica cotidiana, y puestos ubicados en otros espacios (generalmente en cotas altitudinales mayores) con acceso a pasturas y agua ubicados en territorios controlados por grupos sociales, generalmente definidos como familiares. Esta caracterización de un modelo de ocupación del territorio, que es compartida en diferentes sectores de los andes Centro-Sur, fue utilizada para comprender los paisajes culturales de la zona desde el momento del desarrollo de sociedades productivas en el área. En este sentido, la utilización de diferentes herramientas para el estudio del paisaje sirvió para caracterizar y describir esta particularidad, así como también para definir aquellos espacios destinados a la agricultura y sus características tecnológicas y aquellos destinados al pastoreo y sus particularidades arquitectónicas. 
2. Las prácticas cinegéticas no se encuentran exentas de prohibiciones. En relación con las vicuñas, un habitante de La Aguadita, en Antofagasta de la Sierra, comentaba que había días en los que no se podía cazar o que había animales que eran de la Pachamama y, por lo tanto, no podían ser atacados.
Variando la relevancia de la práctica que más importancia tuviera para estas poblaciones, se fueron definiendo paisajes vinculados a aquellos lugares con presencia de recursos hídricos, conformando una dinámica de ocupación del paisaje puneño en base a lugares caracterizados como oasis, donde se podía desarrollar la vida social (Haber, 2006).

La conformación de los grupos sociales que habitaron estos espacios y desarrollaron allí sus modos de vida habría involucrado la intersección de diversas prácticas sociales para poder dar sentido a su reproducción social, en donde la agricultura y el pastoreo eran las actividades centrales, las cuales habrían determinado la estructura espacial del asentamiento. Una manera de entender esta articulación es pensarla en términos de economías campesinas, vinculadas con la reproducción doméstica y con un modo de producción autosustentable (Korstanje, 2007; Quesada, 2007). Dicha visión supone asimismo una forma de vinculación particular con el paisaje y ciertas transformaciones y características del mismo que pueden ser observadas, medidas y registradas por la arqueología, pero también reflexionadas a la hora de entender cómo se construye, reproduce y transforma el paisaje campesino, el cual es entendido como múltiples relaciones entre los diferentes seres que conforman el paisaje (Arnold y Yapita, 1998; Bugallo y Tomasi, 2012; Haber, 2006; Lema, 2013; Rengifo, 1997; Van Kessel y Condori Cruz, 1992).

\section{Recorriendo el paisaje campesino}

La domesticación de animales y plantas implica, entonces, una diferencia significativa en torno a la relación que las poblaciones humanas establecen con el ambiente que las rodea, pero fundamentalmente en la articulación social entre las personas. Claramente, la domesticación de animales permitió que las personas decidieran la reproducción, sacrificio y uso de los recursos de las tropillas, a la vez que disminuían los riesgos que implicaba la obtención de alimento a través de la cacería de especies silvestres (Escola, 1996, 2002; López, 2003, 2006; Olivera, 1997; Olivera et al., 2015; Yacobaccio et al., 1997-1998). Sin embargo, resulta imprescindible imaginar la manera en que esta interacción entre humanos y animales implicó cambios en la relación entre ambos. Hace ya bastante tiempo, algunos autores plantearon que la principal diferencia en torno a la domesticación de animales tenía que ver fuertemente con la negación del aprovechamiento, el cual previamente era asequible libremente, o por lo menos con tabúes impuestos por la cosmovisión local y no por la propiedad individual ${ }^{2}$ (Haber, 2006, 2007; Ingold, 1987, 1988; Vicent García, 1991). Es decir, establecer socialmente y que sea aceptado que un animal que antes podía ser cazado por cualquier persona, estaba ahora bajo el control de un individuo particular, quien podía decidir sobre su muerte y el destino de su carne, lana, cuero, etcétera.

En cierta medida, podemos plantear algo semejante acerca de la agricultura, aunque más vinculado al uso de la tierra, ya que en este caso era necesario apartar una porción de tierra para su producción y cuyos productos finales fueran solamente apropiados por el dueño de dicha parcela. Lo mismo sucede con la gestión del agua, su acopio y su distribución para el riego de las parcelas bajo producción (Barceló, 1988; Quesada, 2007; Vicent García, 1991) e incluso con las semillas y las plantas utilizadas (Lema, 2013). Nuevamente, el cambio en las relaciones sociales es central para comprender el desarrollo de estas prácticas.

Pero superado el proceso inicial de adopción y transformación de las relaciones sociales, la otra gran transformación se ve plasmada en la arquitectura doméstica y productiva, así como también en los cambios de los usos de los distintos espacios. En principio, el aumento de la sedentarización demarca el paisaje a través de estructuras vinculadas con la reproducción doméstica, tales como casas, puestos y refugios, así como 
también espacios como canchones de cultivo y corrales. Esto define materialmente los espacios sociales, creando un monumento (Haber, 2006) en el sentido de marcación y reproducción en el tiempo y que se materializa en el uso de estos espacios. Como vimos antes, generalmente los espacios de fondo de cuenca se asocian a la producción agrícola, mientras que los espacios de vega y pastizales de altura, se plantean como dedicados al pastoreo y eventualmente también a la cacería.

Sin embargo, la reproducción social cotidiana se enmarca en múltiples prácticas que se entretejen para dar sentido a la misma. No es menor el rol relevante que tiene la obtención de agua para el consumo y su gestión comunitaria, la leña, las fuentes de aprovisionamiento para la obtención de materias primas líticas, la cacería de animales silvestres, la obtención de fibras vegetales o recursos vegetales variados para la preparación de medicinas y otras actividades. Todas ellas, además de jugar un rol importante en la reproducción social, definen espacios y vínculos con éstos que requieren ser reflexionados en torno a la construcción y reproducción del paisaje cultural prehispánico.

En el caso particular de este trabajo, queremos poner en consideración este aspecto, articulando diversas escalas de análisis, vinculadas con las diferentes prácticas sobre las cuales hemos trabajado y sobre todo haciendo hincapié en algunos aspectos sobre la construcción y transformación del paisaje en relación con las prácticas sociales realizadas y algunas posibilidades teóricas y metodológicas para su identificación.

\section{Paisajes campesinos en Antofalla}

Como caso de estudio tomaremos las investigaciones llevadas adelante en la quebrada de Antofalla (Figura 1), donde durante varios años se realizaron trabajos de campo en torno a los paisajes culturales y a las características de las ocupaciones humanas durante el primer y segundo milenio de la era (Gastaldi, 2002; Haber, 2006, 2007, 2009; Haber y Lema, 2006; Jofré, 2004; Moreno, 2010; Quesada, 2007).

Esta quebrada está ubicada en el sector noroeste del departamento Antofagasta de la Sierra, provincia de Catamarca, y es una de las quebradas que desciende hacia el Salar de Antofalla, siguiendo el curso de agua que se origina en un par de ojos de agua ubicados a aproximadamente 4.100 y $3.900 \mathrm{~m}$ s.n.m. En su parte más baja forma un cono de deyección con una altura aproximada de $3.400 \mathrm{~m}$ sobre el nivel del mar, donde se pierde en el salar. Esta particularidad del régimen hídrico conforma un paisaje de vega alrededor del curso de agua, mientras que el resto del paisaje es árido. Las características principales de esta área son: clima árido, fuertes variaciones de temperatura entre el día y la noche, baja cobertura vegetal, topografía quebrada, con fuertes pendientes y alturas elevadas. Las precipitaciones son principalmente nivales, con predominancia de las que ocurren en los picos más elevados. La quebrada de Antofalla presenta dos laderas bien marcadas y cuyas pendientes son muy variables, en algunos sectores son muy abruptas mientras que en otros son bastante suaves (Figura 2).

Al indagar acerca del pasado prehispánico de esta quebrada, resalta claramente la presencia de una amplia diversidad de evidencias de prácticas agrícolas, asociadas al recurso hídrico y a los suelos aptos para el desarrollo de estas actividades. En este sentido, una primera escala de análisis apuntó a la evaluación de variables ambientales y la construcción de mapas temáticos que aportaran información relevante en este sentido. En la Figura 3 se pueden observar las áreas con mayor potencial agrícola, situación dada por la presencia de recursos hídricos y suelos aptos para el desarrollo de esta actividad, así como también el análisis de las alturas sobre el nivel del mar, aspecto que constituye de los límites para la producción agrícola en el área. Esto permitió delinear un espacio agrícola de 223 hectáreas donde se observan redes de riego y 


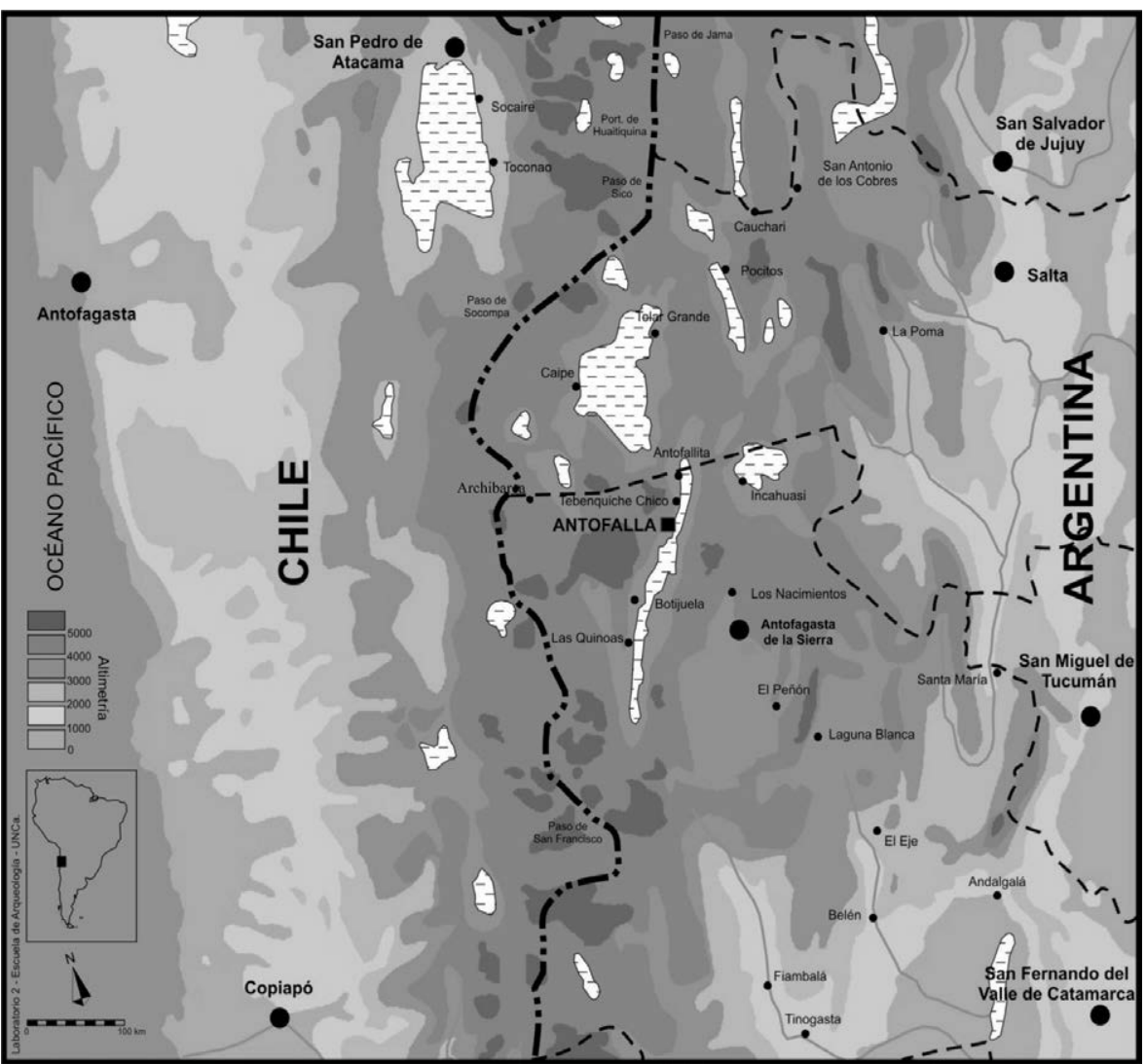

Figura 1. Mapa con la ubicación de la quebrada de Antofalla.

estructuras agrícolas (Quesada, Granizo y Moreno, 2007). Esto supone una superficie máxima potencialmente utilizada para la producción agrícola, aunque algunos sectores pueden haber estado activos mientras otros se encontraban inactivos. Relevamientos posteriores de las redes de riego mostraron que un importante espacio de la quebrada era utilizado para la producción agrícola, vinculado con el uso y distribución del agua y la interpretación de una gestión comunitaria de estos recursos (Quesada, 2007, 2010).

De esta manera, el paisaje agrícola y sus particularidades fueron presentados y analizados, incorporando una matriz histórica de expansión y retracción de estos espacios durante el primer y segundo milenio de la era y las características del manejo del agua a través del estudio del diseño de las redes de riego (Quesada, 2007, 2010; Quesada et al., 2007).

Ahora bien, sabiendo que el pastoreo había sido importante en el paisaje puneño (López, 2003, 2006; Olivera, 1997; Olivera et al., 2015; Yacobaccio et al., 1997-1998), a lo que se suma la información obtenida de conjuntos faunísticos recuperados de algunos sitios del área (Haber, 2006; Moreno, 2010; Revuelta, 2005), resultaba necesario articular el paisaje agrario comentado arriba con los espacios y las transformaciones culturales implicados en la práctica pastoril. En este sentido, resultaba particularmente ineludible analizar e identificar lugares potencialmente utilizados como puestos, a los cuales podrían haber sido trasladados los animales para su alimentación en ciertos momentos del año.

Para ello se articuló una segunda escala de análisis basada tanto en estudios de campo como de laboratorio, la cual implicó la realización de una prospección intensiva y 

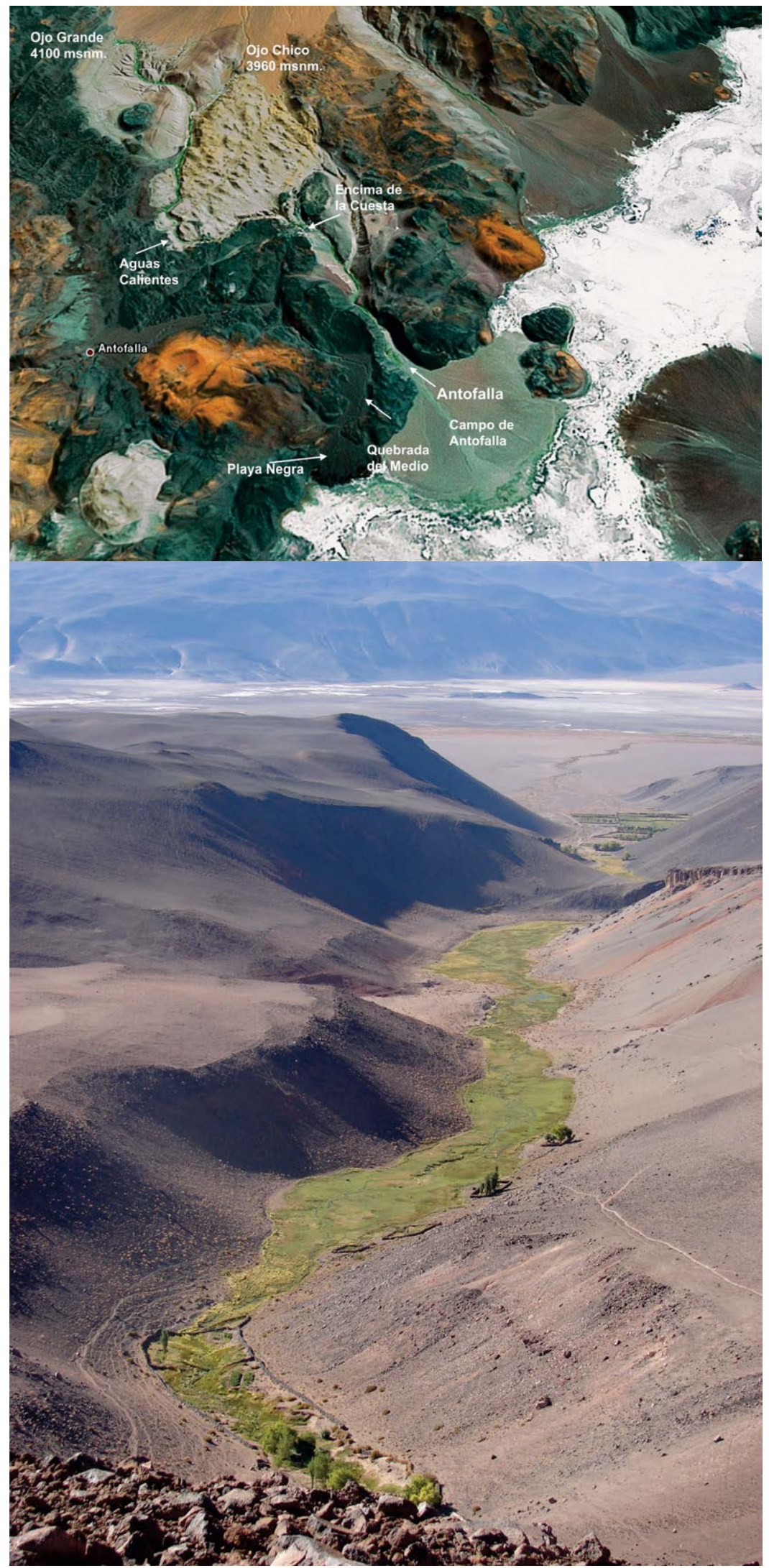

Figura 2. Mapa de la quebrada de Antofalla nombrando diferentes áreas. Abajo vista general de la quebrada, donde se observan las características de ambas laderas y de la presencia de la vega. 
3. Para más información sobre la metodología de prospección y sus particularidades ver Moreno

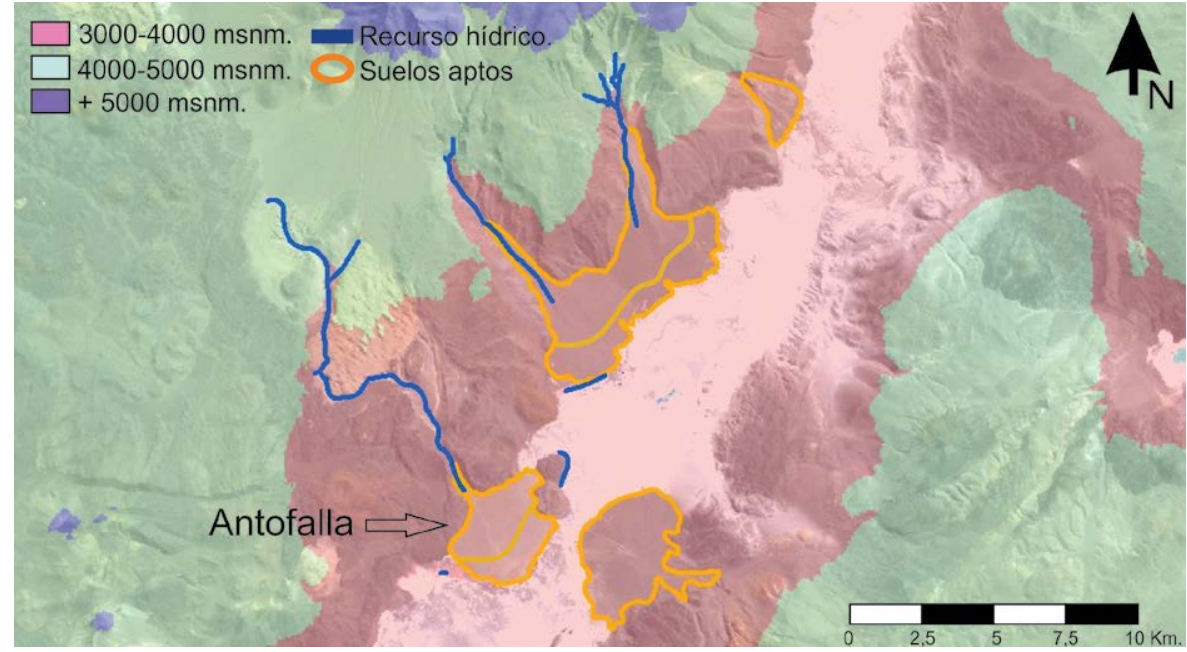

Figura 3. Cartografía del potencial agrícola en Antofalla. Se observan las altitudes sobre el nivel del mar, los suelos aptos para la agricultura y la presencia de recursos hídricos.

sistemática de la totalidad de la quebrada de Antofalla y el posterior análisis de la información obtenida. Dicha prospección fue realizada mediante transectas lineales cada $40 \mathrm{~m}$ y de longitudes variables de acuerdo a las características de la geoforma que se iba recorriendo ${ }^{3}$. La prospección abarcó un área total de $40 \mathrm{~km}^{2}$. Esta metodología permite una excelente relación entre posibilidad de identificación de unidades de registro y tiempo de trabajo en condiciones de alta visibilidad, como son las que se tienen en el paisaje puneño. En estos trabajos de campo se identificaron 3.301 unidades de registro divididas en: estructuras, hallazgos aislados y dispersiones de material en superficie (Moreno, 2010). La prospección brindó información sobre múltiples aspectos de las prácticas sociales prehispánicas en el área. Específicamente en relación al pastoreo, se identificaron algunas estructuras vinculadas con dicha prácticas, tales como refugios y estructuras de reparo, articulados con espacios que podrían haber servido para el resguardo de los pastores. Estas estructuras se ubican en las proximidades de la vega, en sectores del fondo de la quebrada y muchos de ellos son todavía hoy utilizados por los pobladores de Antofalla para observar y cuidar la hacienda.

Sin embargo, el paisaje que se visualizó de manera más clara en este caso fue aquel vinculado a las prácticas de caza, particularmente destinadas a la obtención de vicuñas. La importancia de la caza de vicuñas había sido ya planteada por la información obtenida de los conjuntos arqueofaunísticos de sitios de la quebrada de Tebenquiche Chico, una de las quebradas aledañas a la de Antofalla (Haber, 2006; Moreno, 2010; Revuelta, 2005). En los conjuntos analizados para los sitios Tebenquiche Chico 1 y Tebenquiche Chico 2 pudimos identificar una mayor representación de vicuñas que de llamas, dado que las primeras rondaban el 70 - 80\% del conjunto asignado a camélidos, mientras que las segundas solo alcanzaban el 20 - 30\% del mismo (Haber, 2006; Moreno, 2010; Revuelta, 2005). Ambos sitios poseen ocupación humana durante gran parte del primer milenio de la era y una reocupación durante el período colonial temprano (Haber, 2006; Lema, 2004; Moreno, 2010; Moreno y Lema, 2012; Revuelta, 2005). De igual manera, distintas investigaciones para el área de los Andes Centro-Sur habían hecho hincapié en la importancia de la cacería, particularmente de vicuñas, en ocupaciones humanas durante dicho período histórico (Cartajena, 1994; Haber, 2006; López, 2003, 2006; Moreno, 2010; Olivera, 1997; Yacobaccio y Madero, 1992, entre otros).

Lo que caracterizaba al paisaje de caza en esta área era la articulación de varios aspectos, algunos propios del relieve local y otros vinculados con las transformaciones culturales 
que estructuraban los espacios en los cuales se materializaba una estrategia, que era la de aguardar el paso de las tropillas de animales por aquellos sectores cercanos donde aguardaban, ocultos, los cazadores. Para ello se aprovechaban las características de ambas laderas de la quebrada, construyéndose pequeñas estructuras en forma de arco, denominadas trincheras, que permitían el ocultamiento dada la diferencia de altura entre la ladera y el quiebre de pendiente con la cima (Figura 4). De esta manera, los cazadores podían ocultarse en estos espacios aguardando que las tropillas circularan por estos lugares, siguiendo los movimientos diarios que unen las áreas de pastura y bebedero, en los sectores más bajos de la quebrada, con las áreas donde se ubican los roquedales utilizados como dormideros, en los sectores más altos (Moreno, 2010, 2012; Moreno y Quesada, 2012).

La característica más sobresaliente de este paisaje es la gran cantidad de trincheras (n $=503$ ) que fueron registradas durante la prospección, todas compartiendo aspectos generales más allá de algunas diferencias significativas. Pequeñas estructuras de piedra sin mortero, muchas veces asociadas entre sí, ubicadas en zona elevadas en relación a su entorno directo. La caracterización de este paisaje ha sido objeto de estudio en diversas ocasiones, por lo que aquí no entraremos en detalle (Moreno, 2010, 2012; Moreno y Quesada, 2012). Pero sí haremos una breve referencia a la articulación de diversas herramientas metodológicas para entender algunos aspectos de este paisaje cinegético, las cuales integran una tercera escala de análisis, vinculada con diferentes maneras de registrar el paisaje cinegético y la vinculación de los materiales asociados.

En primer instancia, una de las principales cuestiones que pudimos observar a través de la utilización de herramientas técnicas para el estudio del paisaje cinegético, fue la presencia de estas estructuras a lo largo de prácticamente toda la quebrada, más allá de la ausencia en algunos sectores muy puntuales y la mayor abundancia en otros (Figura 5). Otro aspecto que se pudo observar es que las trincheras se ubicaban en espacios elevados. Para ello, se emplearon no sólo las fotografías realizadas en el campo y el registro de las características de la ubicación en las planillas de campo, sino también la observación en 3D (Figura 6) y el relevamiento topográfico con las ubicaciones de las trincheras (Moreno, 2010). Allí notamos la forma en que estas estructuras se ubicaron en los sectores más elevados de la unión entre ladera y quiebre de pendiente de la cima o su vinculación directa con rasgos topográficos relevantes para aumentar el ocultamiento por parte de los cazadores. Realizamos algunos análisis cuantitativos para evaluar dicha situación, notándose una mayor presencia de estas estructuras en el sector de cima (74\% del total de estructuras identificadas). Sin embargo, incluso aquellos casos en los que se ubicaban en los sectores más deprimidos de la quebrada, siempre se encontraban levemente elevadas en relación con el entorno directo. De esta manera, se obtiene la posibilidad de ocultarse de los animales, ubicados en sectores más bajos, sin la necesidad de construir estructuras de gran tamaño, que inclusive pudiesen llamar la atención de las presas.

Pero la relevancia de las prácticas de caza no solo fue observada a partir de las estructuras construidas sino también por la presencia de un importante conjunto de puntas de proyectil. Estos instrumentos fueron analizados con el objetivo de construir una cronología para los mismos comparando con registros de otros lugares, y así evaluar los distintos sistemas de armas utilizados y las materias primas utilizadas (Moreno, 2010, $2011,2016)$. A través de un análisis tecnológico-funcional, sumado a la evaluación de las características del diseño, de su asignación funcional a un determinado tipo de arma y su comparación con piezas de contextos semejantes ubicados cronológicamente (Aschero, 1975, 1983, 1988; De Souza, 2004; Escola, 1987, 2000; Hocsman, 2006; Martínez, 2003; Ratto, 1994, 2003; Shott, 1997) pudimos construir una cronología relativa de los paisajes cinegéticos. El número total de puntas de proyectil registradas en la prospección fue de 192, al tiempo que 13 de las cuales se encontraban completas y 179 fracturadas. En el caso de estas últimas, a veces se registra una representación alta de la pieza y en otros 

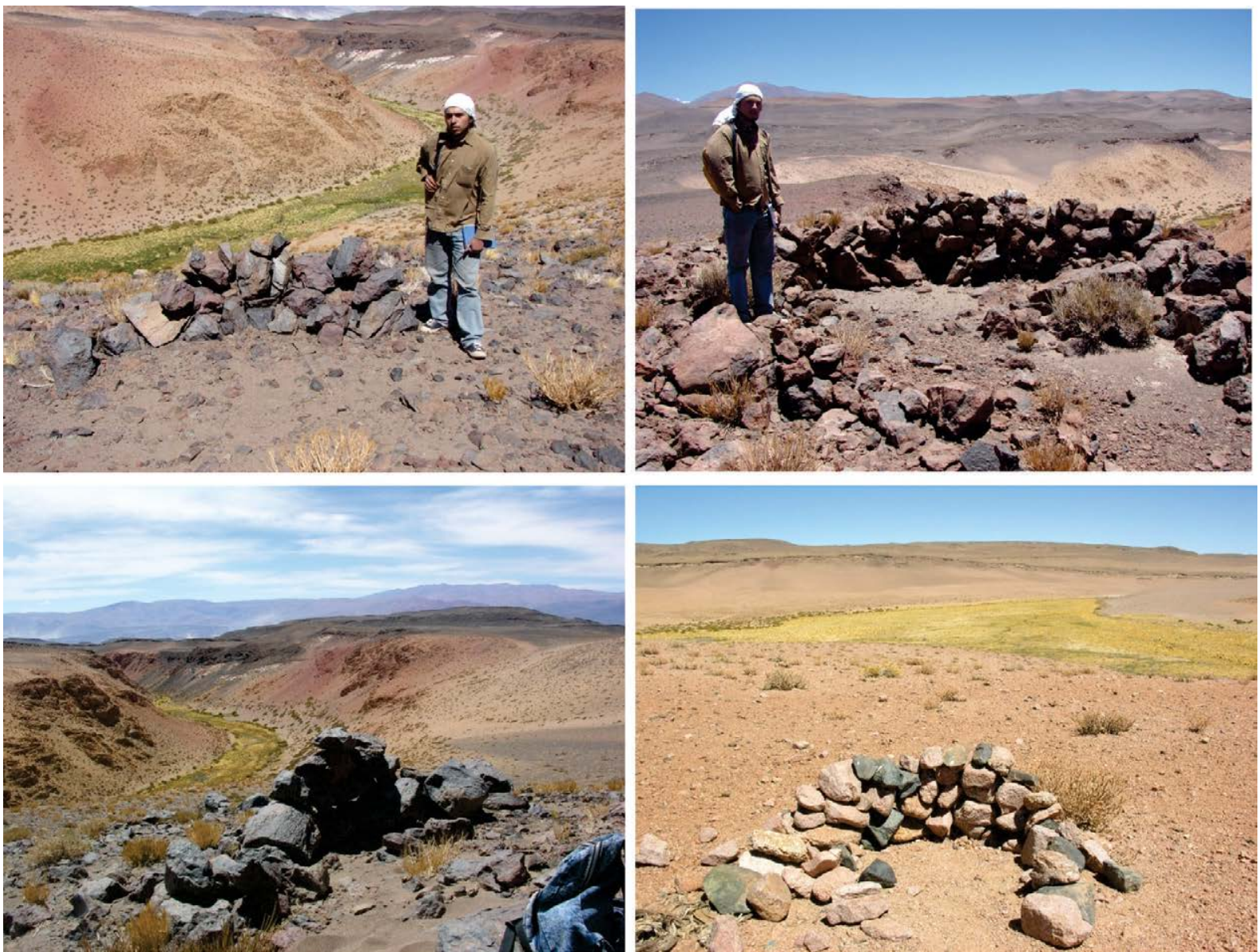

Figura 4. Trincheras identificadas en la quebrada de Antofalla. Nótese la ubicación elevada de las mismas en relación al entorno directo.

casos sólo se destaca la presencia de fragmentos muy pequeños. Con respecto a las materias primas utilizadas, el 90\% del conjunto se encuentra manufacturado en basaltoandesita negro y en obsidiana, situación similar a lo observado en otros conjuntos de la zona (Moreno, 2005). La primera de estas materias primas, que fue utilizada para la manufactura del 65,5\% del conjunto total, proviene de la fuente de aprovisionamiento que se encuentra en la desembocadura de la quebrada de Antofalla. Por otro lado, en el caso de la obsidiana se presentan dos variedades: una semejante a la de Ona, ubicada a unos $15 \mathrm{~km}$ de la quebrada de Antofalla y otra con mayor opacidad que podría provenir de Archibarca, donde fueron registrados nódulos con características similares (Haber, 2003) (Figura 7). Por lo tanto, se observa que se aprovecharon materias primas presentes localmente principalmente, y en segundo término se utilizó la obsidiana de Ona, la cual se ubica a una distancia considerable pero relativamente cercana si comparamos la distribución de esta materia prima en el NOA (Escola, Glascock, Korstanje y Sentinelli, 2009; Lazzari, 1997, 2010; Yacobaccio, Escola, Pereyra, Lazzari y Glascock, 2004).

Para la vinculación de las puntas de proyectil con las características del paisaje de caza, se realizaron dos análisis que luego permitíeron evaluar su distribución espacial en relación con las trincheras; por un lado la asignación funcional de estos instrumentos a distintos tipos de armas y, por otro, su correlación cronológica. Ambos análisis perseguían el objetivo de historizar el paisaje cinegético evaluando diferencias en la distribución de las puntas de proyectil. En el caso de la asignación funcional y considerando distintas herramientas metodológicas (Ratto, 1994, 2003; Shott, 1997), se logró asignar un total de 38 puntas de proyectil a un tipo de arma (19,8\%), de las cuales 


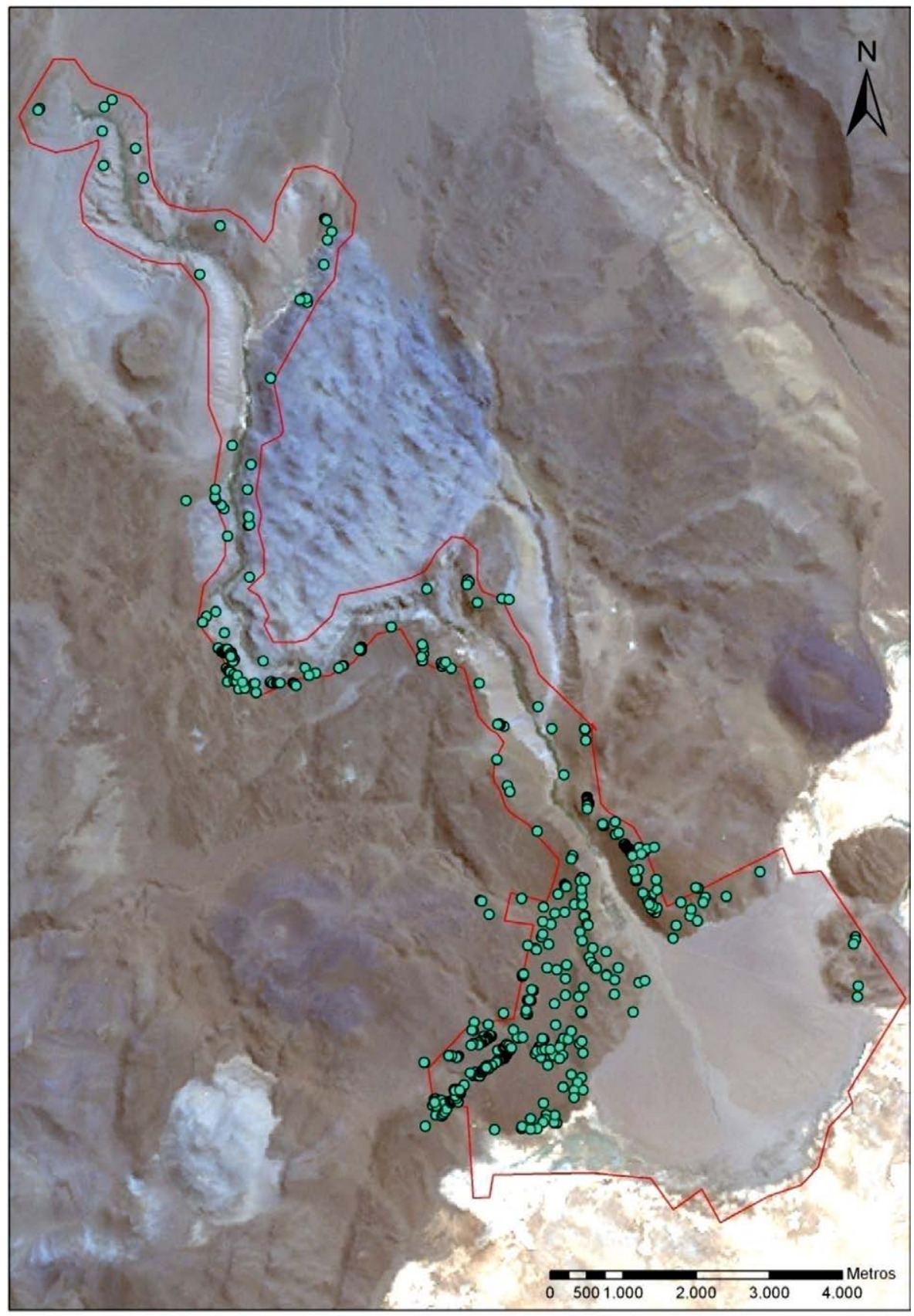

Figura 5. Distribución de las trincheras en la quebrada de Antofalla.

diez fueron asignadas a arco y flecha, tres a armas de mano, tres a lanza arrojadiza y 22 a dardos de lanzadera. Por otro lado, la comparación de los diseños con aquellos especímenes ubicados cronológicamente en otras regiones - principalmente en la microrregión de Antofagasta de la Sierra (Hocsman, 2006; Martínez, 2003) - permitió observar ejemplares que se ubican en distintos momentos históricos pero que a la vez refieren a un largo término de uso de estos espacios. En la Tabla 1 se observan estas características y su correlación cronológica. Para simplificar la información aquí expuesta, existen tres grandes períodos o momentos: ocupaciones tempranas que se correlacionan con diseños ubicados ca. 8600 años AP, un momento de transición entre el 5150 y el 3430 años AP y un último momento correspondiente al primer y segundo milenio de la era ${ }^{4}$.

4. Para más información sobre las características de las puntas de proyectil y la tecnología de caza ver Moreno (2010, 2011, 2016). 


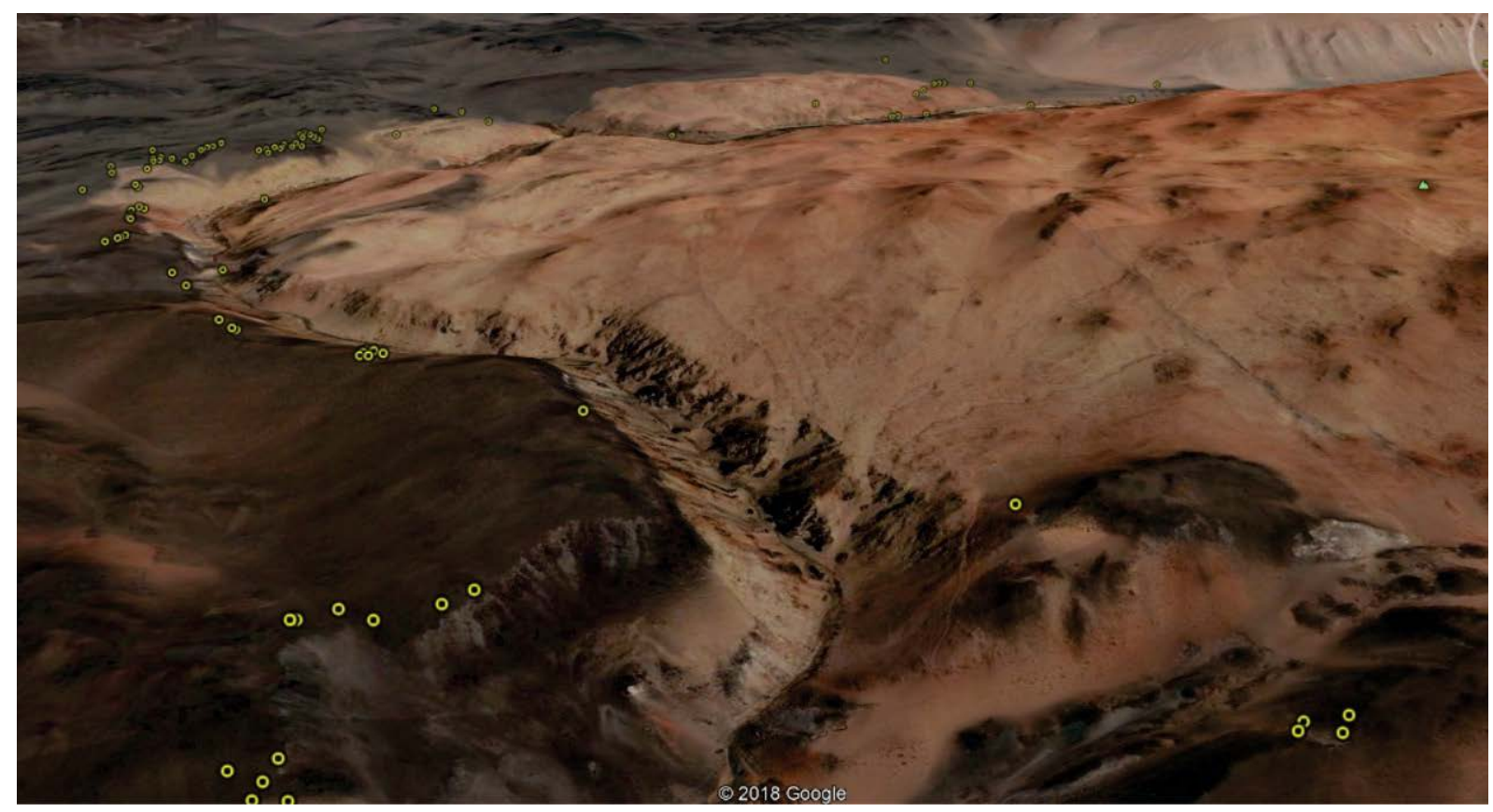

Figura 6. Vista en 3D de un sector de la quebrada de Antofalla, donde se observa la ubicación en los sectores elevados de las trincheras.

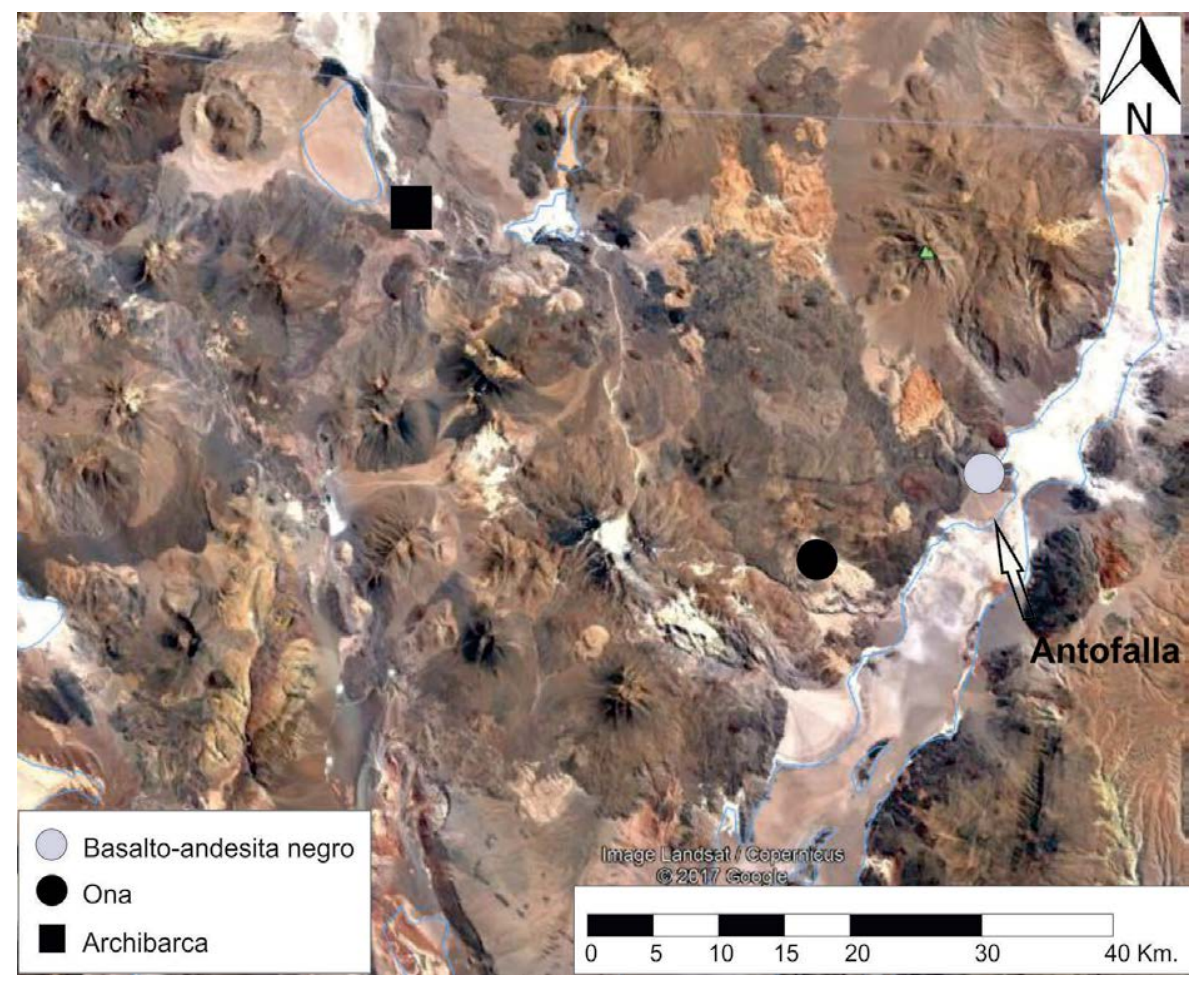

Figura 7. Ubicación de las fuentes de aprovisionamiento de las materias primas utilizadas para la manufactura de puntas de proyectil.

Ahora bien, esta información serviría, tal como dijimos antes, para evaluar diferencias en el uso del paisaje por parte de las poblaciones a través del tiempo o de las características de las armas. De esta forma, correlacionar las trincheras con esta información de las puntas de proyectil podría servir para generar una historia del paisaje cazador. Sin 


\begin{tabular}{|c|c|c|}
\hline Tipo & Semejanza & Período \\
\hline Af-I & Peñas Chicas E & 4150 - 3430 años AP \\
\hline Af-II & Quebrada Seca A - Tambillo-1 & $8600 \mathrm{AP}$ \\
\hline Af-III & - & - \\
\hline Af-IV & Peñas Chicas C & $4150-3430$ años AP \\
\hline Af-V & - & - \\
\hline Af-VI & Quebrada Seca F & $4150-3430$ años AP \\
\hline Af-VII & Peña de la Cruz A & 7270 años AP \\
\hline Af-VIII & Peñas Chicas A & $4150-3430$ años AP \\
\hline Af-IX & $\begin{array}{l}\text { Registros semejantes en TC1, Casa Chávez } \\
\text { Montículos, Chaschuil, Real Grande I, Tulan-54 }\end{array}$ & $1^{\circ}$ Milenio d.C. \\
\hline Af-X & - & - \\
\hline Af-A & Quebrada Seca B & 7350 - 3500 años AP \\
\hline Af-B & - & - \\
\hline Af-C & - & - \\
\hline Af-D & Peñas Chicas 4 & 4150 - 3430 años AP \\
\hline
\end{tabular}

Tabla 1. Asignación cronológica de las puntas de proyectil en comparación con diseños de otros sitios del área.

embargo, tal como observamos en la Figura 8 , notamos que no se observan patrones claros de diferenciación en la distribución de las puntas de proyectil por ambas variables, lo que implicaría que los mismos sectores fueron utilizados a través del tiempo y a pesar de los cambios funcionales de las armas, lo que supone una suerte de continuidad en el tiempo de esta práctica, por lo menos en torno a los espacios utilizados.

A pesar de la presencia de puntas de proyectil correspondientes al primer milenio de la era, e incluso cartuchos de bala, en distintos sectores de la quebrada de Antofalla, la primera interpretación habría sido la de asignar la infraestructura vinculada con la cacería a momentos históricos correspondientes a períodos tempranos. Sin embargo, los registros faunísticos de los sitios excavados en la zona han mostrado muy altos porcentajes de camélidos silvestres, superiores a aquellos asignados taxonómicamente a domesticados (Haber, 2006; Moreno, 2010; Revuelta, 2005). Al mismo tiempo, en otros sitios de los Andes Centro-Sur se muestra la importancia de esta práctica para momentos correspondientes al primer milenio e incluso al segundo milenio de la era (Cartajena, 1994; López, 2003, 2006; Olivera, 1997; Yacobaccio y Madero, 1992).

Por lo tanto, asumiendo la importancia de la cacería en estos momentos históricos pero también la presencia de espacios destinados a la agricultura, las unidades domésticas establecidas y los rebaños de animales domésticos, resulta relevante reflexionar acerca de los espacios en que se habrían dado los encuentros entre cazadores y vicuñas. En un modelo que prioriza lo altitudinal para construir los usos del paisaje, las prácticas de caza se habrían realizado en los sectores elevados de las quebradas, que son los espacios donde se ubican actualmente los territorios de las vicuñas. Sin embargo, los registros recuperados en las prospecciones permiten interpretar que las prácticas de cacería no habrían sido realizadas en espacios alejados, como podrían ser las partes altas de la quebrada, sino simplemente aquellos espacios que no eran frecuentados por las poblaciones humanas para la agricultura o para la alimentación de los rebaños de llamas $\mathrm{y}$ donde las vicuñas pudiesen alimentarse y beber agua, sin estar frente al peligro que 


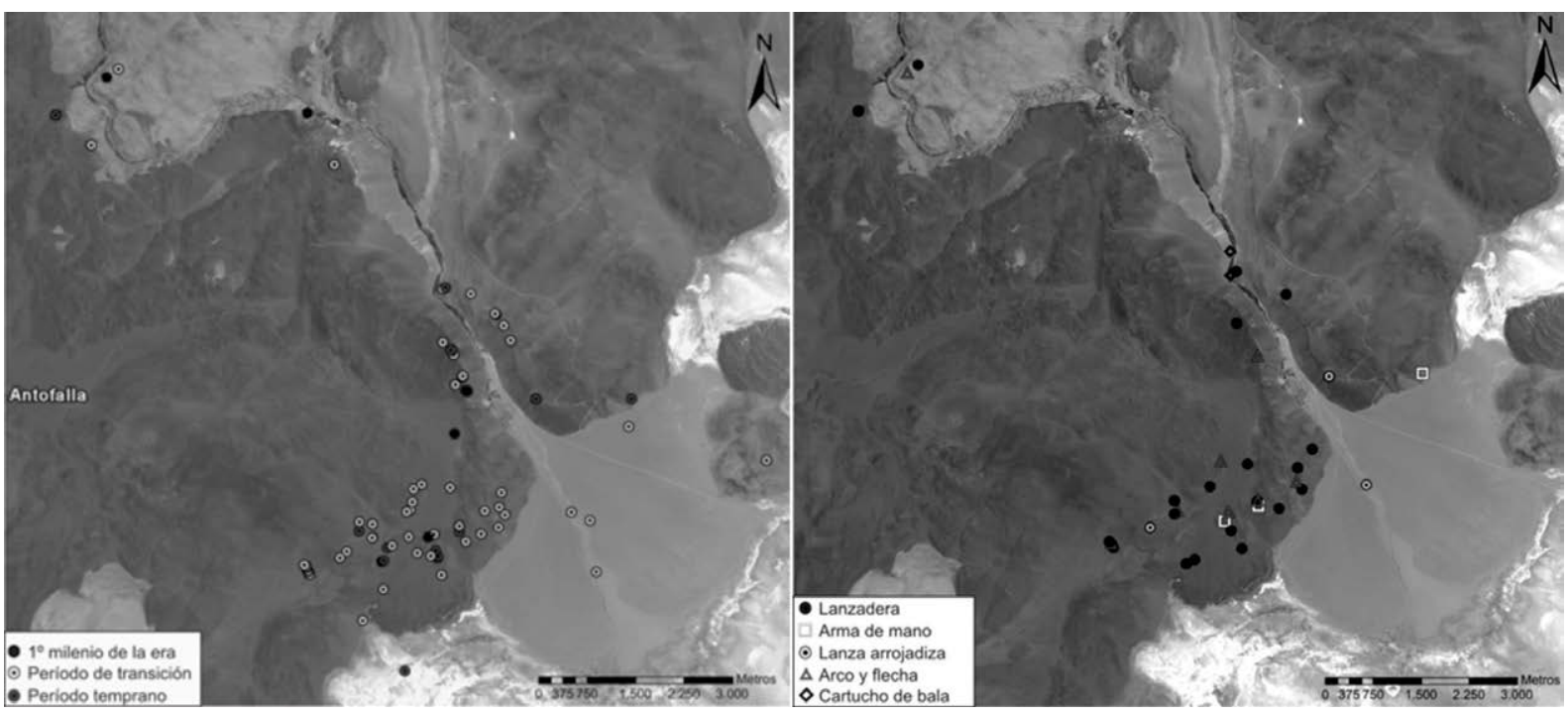

Figura 8. Distribución de las puntas de proyectil de acuerdo a su asignación a distintos tipos de armas (derecha) y a su asignación cronológica (izquierda).

representaban las personas circulando constantemente por allí. Actualmente, hemos observado tropillas de vicuñas utilizando algunos sectores de la quebrada de Antofalla, los cuales no suelen ser visitados por los pobladores. Por lo tanto, espacios como los de Playa Negra, Aguas Calientes y Ojo de Agua (ver Figura 2) entre otros, habrían podido ser utilizados como lugares de cacería durante el primer milenio de la era, reutilizando antiguas trincheras para realizar las cacerías.

Luego, esta imagen permite reflexionar sobre un paisaje campesino, en donde habrían interactuado prácticas como el pastoreo, la agricultura y la cacería dando forma a la reproducción social de las personas que allí vivieron. Sin embargo, existen otros recursos que conforman el paisaje campesino y son relevantes para la reproducción social. Uno de ellos podría haber sido la materia prima lítica, particularmente aquí haremos referencia a la obsidiana de Ona.

Esta materia prima tuvo una gran relevancia en el Noroeste Argentino, ya que se encuentra presente en sitios muy distantes, tal como se observa en distintos análisis realizados con muestras provenientes de algunos lugares ubicados a más de 400 $\mathrm{km}$ desde la fuente de aprovisionamiento (Escola et al., 2009; Lazzari, 1997, 2010; Yacobaccio et al., 2004). La quebrada de Antofalla se ubica a pocos kilómetros de esta cantera -aproximadamente $15 \mathrm{~km}$ - y fue aprovechada para la manufactura de instrumentos líticos, principalmente puntas de proyectil (Moreno, 2005, 2011). Pero a pesar de esta distancia relativamente corta en relación con la escala más amplia de distribución de esta materia prima, el traslado hasta Ona para seleccionar y recolectar nódulos y núcleos habría implicado ciertos cambios o articulaciones particulares en torno a las rutinas cotidianas de estas poblaciones. Aunque quizás también haya sido un recurso relevante para pensar la articulación a una escala mucho mayor del paisaje, la cual incorporase la interacción social basada en el intercambio y la circulación de esta materia prima por parte de estas poblaciones con grupos de zonas lejanas y ambientes con ofertas de recursos muy diferentes (Chaparro y Moreno, 2010; Escola, et al., 2009; Giesso, 2003; Lazzari, 1997, 2010; Yacobaccio et al., 2004).

Otro recurso central para comprender la reproducción doméstica y la construcción del paisaje campesino es la leña. En un ambiente donde este sería un recurso relativamente escaso, su selección, búsqueda y acopio resulta un aspecto destacado. Actualmente, en 
el caso de Antofalla, la población realiza al menos dos salidas de procuración de leña al mes y se suma a aquella que se puede obtener en recolecciones realizadas en traslados a otras quebradas u otros lugares para llevar animales para pastorear. Según Jofré (2004) las especies más utilizadas son la "rica" (Acantholippia punensis) y la "tramontana" (Ephedra breana) como leñas para la combustión destinada a la cocción de alimentos, mientras que la "chacha" (Parastrephia quadrangularis) es utilizada en rituales domésticos para sahumar, como por ejemplo en el caso de la señalada o en corpachadas para el primero de agosto, día de los muertos, etcétera. La áreas de recolección de estos recursos se ubican generalmente en torno a los espacios de control de las distintas unidades domésticas y en derredor de los corredores utilizados por estas unidades para el traslado de hacienda a los puestos (Jofré, 2004). Por lo tanto, ya sea en torno a espacios cercanos a la quebrada de Antofalla o a lugares más alejados vinculados con el tránsito hacia los puestos, los espacios destinados a la obtención de leña conforman una parte vital para la reproducción doméstica y la construcción de este paisaje social.

Entonces, lo que he intentado mostrar hasta aquí es que más allá de la relevancia de distintas prácticas sociales para las poblaciones campesinas que habitaron la quebrada de Antofalla, hay algunas cuya materialidad es más visible y por lo tanto son las que predominan en las interpretaciones y en la construcción de las cartografías específicas. Sin embargo, si profundizamos la caracterización de la interacción entre poblaciones locales y el paisaje cultural, comenzamos a notar múltiples prácticas que definen y marcan el territorio. Así, podemos imaginar en el área de Antofalla un paisaje demarcado, construido y reproducido por la articulación de diferentes prácticas sociales y esencialmente por la vinculación entre los distintos seres que conformaron y conforman este lugar. De igual manera, vemos cómo la integración de diferentes escalas de análisis que implican prospecciones, excavaciones, teledetección, entrevistas con pobladores locales, recorridos por el territorio con estas personas, etc., permiten articular e incluso visualizar el paisaje desde múltiples miradas. De esta manera, se puede pensar en un mapa de la quebrada de Antofalla y zonas cercanas delimitado y definido por estas prácticas sociales. Sin embargo, construir esta cartografía resulta imposible, si no es simplemente delimitando todo aquel territorio utilizado para dar sentido a la reproducción social de los habitantes de Antofalla a través del tiempo.

\section{Cartografías, paisajes y prácticas sociales}

En este trabajo partimos de la idea de caracterizar y evaluar la forma en que se vinculan las prácticas sociales con diferentes espacios de la quebrada de Antofalla y retomar la importancia que tiene esta interacción para entender la dinámica de reproducción social de este lugar a través del tiempo. Para ello, partimos inicialmente de la idea de no centrarnos únicamente en aquellas prácticas que suelen ser predominantes a la hora de interpretar los paisajes culturales puneños, particularmente el pastoreo y la agricultura. Esto no significa negar dichas prácticas o no tener en cuenta su importancia para las poblaciones locales, sino que más bien la intención es la de considerar las múltiples prácticas sociales que dan sentido a la reproducción social de estas poblaciones y, particularmente, evaluar algunas escalas de análisis para estudiar la forma en que estas demarcan y constituyen el paisaje.

Por ello, comenzamos mostrando aquellos aspectos vinculados con la agricultura y el pastoreo en Antofalla, analizando la presencia de canchones de cultivo, redes de riego, refugios y otros elementos vinculados con estas dos prácticas y sus implicancias en la construcción del paisaje local. Vale recalcar que además, hoy en día, estas dos actividades son las que se encuentran presente en las lógicas de reproducción de la población y que demarcan el paisaje cultural y definen las características principales de la reproducción social. 
Sin embargo, nos interesaba pensar en aquellas otras prácticas sociales que también eran aspectos importantes de la reproducción social de estas poblaciones, que dejaban marcas en el territorio y que definían un paisaje social en el área. La prospección en Antofalla permitió conocer varios aspectos relevantes acerca de estas prácticas, aunque las de cacería fueron las que mayores evidencias presentaron. Trincheras, puntas de proyectil, escondites y las evidencias provistas por los conjuntos faunísticos indicaron la importancia de esta actividad y permitieron interpretar la construcción de un paisaje cinegético en el que los cazadores anticipaban los movimientos de las presas para aumentar las posibilidades del éxito al obtener blancos cercanos.

Pero también nos interesamos por otras prácticas que demarcaron el paisaje, tales como la obtención de materias primas para la manufactura de instrumentos líticos o la búsqueda de leñas, como algunas de las tantas prácticas que permiten la reproducción social y que constituyen paisajes culturales vividos, experimentados y reproducidos a través del tiempo.

Por lo tanto, resulta claro que los SIG y otras herramientas de gestión y análisis del paisaje son importantes vehículos para el estudio y la interpretación de las historias de ocupación y transformación del territorio por parte de las poblaciones humanas. A través de ellos, es posible visualizar espacios destinados a distintas prácticas, caracterizar los espacios y sus usos, generar modelos de construcción de territorios a través del tiempo y poder divulgar estos conocimientos a distintos ámbitos, inclusive en las comunidades donde se desarrollan nuestras actividades. En este sentido, los SIG presentan varias ventajas que se refieren a la posibilidad de visualizar esta variabilidad y generar mapas temáticos tales como los que mostramos en este artículo y en otros trabajos del presente dossier. En este sentido, poner en marcha diversas escalas de trabajo tanto a nivel más general, tales como teledetección y análisis de pendientes o relieves, como en el caso de análisis particulares, como pueden ser los materiales recuperados de la prospección e incluso la consulta y aprendizaje con los pobladores actuales del lugar, pretendió ser unos de los aportes de este trabajo, particularmente haciendo hincapié en la posibilidad de pensar diferentes maneras de observar las prácticas sociales a partir de estas múltiples escalas de análisis.

Sin embargo, es necesario que seamos conscientes de la manera en que estas herramientas pueden sesgar las construcciones de los territorios campesinos y la posibilidad de visualizar otras prácticas sociales centrales para estas poblaciones, intentando no caer en ciertos determinismos ambientales acerca de la interacción entre los humanos y el entorno que los rodea. Por supuesto, queda mucho camino por recorrer en esta reflexión pero aquí pretendíamos por lo menos generar un espacio que nos permita observar la importancia de las herramientas analíticas para el estudio del paisaje, apoyado además en preguntas que nos lleven a tratar de enfocarnos en un paisaje entendido en términos múltiples, dinámicos y transformables en la continua interacción entre los seres que participan de este entramado.

\section{Agradecimientos}

Las prospecciones fueron realizadas en Antofalla con subsidios de la Secretaría de Ciencia y Técnica de la Universidad Nacional de Catamarca (SECyT-UNCA), la Agencia Nacional de Promoción Científica y Tecnológica (ANPCyT) y el Consejo Nacional de Investigaciones Científicas y Tecnológicas (CONICET) a través de proyectos de investigación dirigidos por el Dr. Alejandro Haber. Quiero agradecer a todas las personas que participaron de las prospecciones y que de una u otra manera ayudaron a reflexionar sobre esta temática. También expreso mi agradecimiento a los evaluadores del artículo cuyos comentarios permitieron enriquecer el contenido del mismo. Finalmente a los pobladores de Antofalla por permitirnos aprender tanto de ellos en esos años de trabajo. 


\section{Referencias citadas}

" Aldenderfer, M. y Maschner, H. (Eds.). (1996). Anthropology, Space, and Geographic Information Systems. Oxford: Oxford University Press.

» Anschuetz, H., Wilshusen, R. y Scheick, C. (2001). An archaeology of landscapes: Perspectives and directions. Journal of Archaeological Research, 9(2), 152-197.

» Arnold, D. y Yapita, J. (1998). Río de vellón, río de canto. Cantar a los animales, una poética andina de la creación. La Paz: Hisbol/Instituto de Lengua y Cultura Aymara.

» Aschero, C. (1975). Ensayo para una clasificación morfológica de artefactos líticos aplicada a estudios tipológicos comparativos. Informe al CONICET. Manuscrito inédito.

" Aschero, C. (1983). Ensayo para una clasificación morfológica de artefactos líticos. Apéndice A y B. Cátedra de Ergología y Tecnología, Facultad de Filosofía y Letras, Universidad de Buenos Aires. Manuscrito inédito.

" Aschero, C. (1988). De punta a punta: producción, mantenimiento y diseño en puntas de proyectil precerámicas de la Puna argentina. En Precirculados de las ponencias científicas. IX Congreso Nacional de Arqueología Argentina (pp. 219-229). Buenos Aires: Universidad de Buenos Aires.

" Baena Preysler, J., Blasco Bosqued, C. y Quesada Sanz, F. (Eds.). (1997). Los SIG y el Análisis Espacial en Arqueología. Madrid: Universidad Autónoma de Madrid.

» Barceló, M. (1988). La arqueología extensiva y el estudio de la creación del espacio rural. En M. Barceló, H. Kirchner, J. Lluró, R. Martí y J. Torres (Eds.), Arqueología medieval en las afueras del «medievalismo» (pp. 195-274). Barcelona: Crítica.

» Bruno, D. y Thomas, J. (2008). Handbook of Landscape Archaeology. California: Left Coast Press.

" Bugallo, L. y Tomasi, J. (2012). Crianzas mutuas. El trato a los animales desde las concepciones de los pastores puneños (Jujuy, Argentina). Revista Española de Antropología Americana, 42(1), 205-224.

»Cartajena, I. (1994). Determinación de restos óseos de camélidos en dos yacimientos del Loa Medio (II Región). Estudios atacameños, 11, 25-52.

"Cladera, J. (2013). Pessoas que Cruzam Territórios e Territórios que são Pessoas - As Experiências do Direito Espacial nos Andes: um estudo de caso no noroeste argentino. ILHA Revista de Antropología, 15(1), 149-178.

»Criado Boado, F. (1999). Del terreno al espacio: planteamientos y perspectivas para la arqueología del paisaje. CAPA, 6, 1-82.

»Curtoni, R. (2007). Arqueología y paisaje en el área centro-este de La Pampa. (Tesis Doctoral inédita), Universidad Nacional de La Plata, Argentina.

》De Souza, P. (2004). Tecnologías de proyectil durante los períodos Arcaico y Formativo en el Loa Superior (Norte de Chile) a partir del análisis de puntas líticas. Chungara. Revista de Antropología Chilena, Volumen especial(1), 61-76.

"Delfino, D. (2001). Of pircas and the limits of society: ethnoarchaeology in the la Puna, Laguna Blanca. Catamarca. Argentina. En L. Kuznar (Ed.), Ethnoarchaeology of Andean South America: Contributions to Archaeological Method and Theory, (pp. 116-137). Ann Arbor: International Monographs in Prehistory. 
" Di Lullo, E. (2010). El espacio residencial durante el 1er milenio d.C. en la Quebrada de Los Corrales (El Infiernillo, Tucumán). (Tesis de Licenciatura inédita), Universidad Nacional de Tucumán, Argentina.

"Diamond, J. (2002). Evolution, consequences and future of plant and animal domestication. Nature, 418, 700-707.

»Escola, P. (1987). Las puntas de proyectil del formativo en Puna y Quebradas de Acceso: un estudio tecno-tipológico de cuatro casos de análisis. (Tesis de Licenciatura inédita), Universidad de Buenos Aires, Argentina.

»Escola, P. (1996). Riesgo e incertidumbre en economías agro-pastoriles: consideraciones teórico-metodológicas. Arqueología, 6, 9-24.

»Escola, P. (2000). Tecnología lítica y sociedades agropastoriles tempranas. (Tesis Doctoral inédita), Universidad de Buenos Aires, Argentina.

"Escola, P. (2002). Caza y pastoralismo: un reaseguro para la subsistencia. Relaciones de la Sociedad Argentina de Antropología, XXVII, 233-245.

"Escola, P., Glascock, M., Korstanje, M. y Sentinelli, N. (2009). Laguna Cavi y El Médano: obsidianas en circulación caravanera. En O. Palacios, C. Vásquez, T. Palacios y E. Cabanillas (Eds.), Arqueometría Latinoamericana: Segundo Congreso Argentino y Primero Latinoamericano (volumen 1) (pp. 103-108). Buenos Aires: Comisión Nacional de Energía Atómica (CNEA).

» Figuerero Torres, M. J. e Izeta, A. (Eds.) (2013). El uso de Sistemas de Información Geográfica (SIG) en arqueología sudamericana. Oxford: BAR International Series 2497, Archaeopress.

»Franco Salvi, V. y Berberián, E. (2011). Prácticas Agrícolas de Sociedades Campesinas en el Valle de Tafí (100 a.C.- good.C.). Revista Chilena de Antropología, 24, 119-145.

" García Sanjuán, L. (2005). Introducción al Reconocimiento y Análisis Arqueológico del Territorio. Barcelona: Ariel.

" Gastaldi, M. (2002). Tecnología y sociedad: biografía e historia social de las palas del oasis de Tebenquiche Chico. (Tesis de Licenciatura inédita), Universidad Nacional de Catamarca, Argentina.

" Giesso, M. (2003). Stone tool production in the Tiwanaku Hearthland. En A. Kolata (Ed.), Tiwanaku and its Hinterland: Archaeological and Paleoecological Investigations in the Lake Titicaca Basin of Bolivia (Volumen 2) (pp. 363-383). Washington DC: Smithsonian Institution Press.

" Gillings, M. (2012). Landscape Phenomenology, GIS and the Role of Affordance. Journal of Archaeological Method and Theory, 19, 601-611.

» Göbel, B. (2002). La arquitectura del pastoreo: Uso del espacio y sistema de asentamientos en la Puna de Atacama (Susques). Estudios Atacameños, 23, 53-79.

" Haber, A. (2003). Hunting after Domestication. Trabajo presentado en Conference of Hunting and Gathering Societies 9. Edimburgo, Escocia.

"Haber, A. (2006). Una arqueología de los oasis puneños. Domesticidad, interacción e identidad en Antofalla. Primer y segundo milenios d.C. Córdoba: Universitas/Jorge Sarmiento.

"Haber, A. (2007). Arqueología de uywaña. Un ensayo rizomático. En A. Nielsen, C. Rivolta, V. Seldes, M. Vázquez y P. Mercolli (Comp.), Producción y circulación prehispánicas de bienes en el Sur Andino (pp. 13-36). Córdoba: Brujas.

" Haber, A. (2009). Animism, Relatedness, Life: Post-Western Perspectives. Cambridge Archaeological Journal, 19(3), 418-430. 
» Haber, A. y Lema, C. (2006). La pura opinión de Vladimiro Weisser y la población indígena de Antofalla en la Colonia Temprana. Intersecciones en Antropología, 7, 179191.

» Harris, D. (1996). Domesticatory Relationships of People, Plants, and Animals. En R. Ellen y K. Fukui (Eds.), Redefining Nature. Ecology, Culture, and Domestication (pp. 437463). Oxford: Berg.

» Hocsman, S. (2006). Producción lítica, variabilidad y cambio en Antofagasta de la Sierra ca. 5500 - 1500 AP-. (Tesis Doctoral inédita), Universidad Nacional de La Plata, Argentina.

» Ingold, T. (1987). The appropriation of nature. Essays of human ecology and social relations. lowa: University of lowa Press.

» Ingold, T. (1988). Introduction. En T. Ingold (Ed.), What is an Animal? (pp. 1-16). Londres: Unwin Hyman.

» Ingold, T. (1993). The temporality of the landscape. World Archaeology, 25(2), 152-174.

»Jofré, I. (2004). Arqueología del fuego. Tebenquiche Chico. (Tesis de Licenciatura inédita), Universidad Nacional de Catamarca, Argentina.

» Korstanje, A. (2007). Territorios campesinos. Producción, circulación y consumo en los Valles Altos. En A. Nielsen, M. Rivolta, V. Seldes, M. Vázquez y P. Mercolli (Comp.), Producción y circulación prehispánica de bienes en el sur Andino (pp. 191-223). Córdoba: Editorial Brujas.

» Korstanje, A., Quesada, M., Franco Salvi, V., Lema, V. y Maloberti., M. (2015). Gente, tierra, agua y cultivos. Los primeros paisajes agrarios del Noroeste Argentino. En A. Korstanje, M. Lazzari, M. Basile, F. Bugliani, V. Lema, L. Pereyra Domingorena y M. Quesada (Eds.), Crónicas Materiales Precolombinas. Arqueología de los primeros poblados del Noroeste Argentino (pp. 721-749). Buenos Aires: Sociedad Argentina de Antropología.

» Korstanje, A. y Yacobaccio, H. (2007). Los procesos de domesticación vegetal y animal. Un aporte a la discusión argentina en los últimos 70 años. Relaciones de la Sociedad Argentina de Antropología, XXXII, 191-215.

» Kvamme, K. (1999). Recent Directions and developments in geographical information systems. Journal of Archaeological Research, 7(2), 153-201.

»Lazzari, M. (1997). La economía más allá de la subsistencia: intercambio y producción lítica en el Aconquija. Arqueología, 7, 9-50.

» Lazzari, M. (2010). Landscapes of circulation in NW Argentina: the workings of obsidian and ceramics during the first millennium AD. En A. Agbe-Davis y A. Bauer (Eds.), Social Archaeologies of Trade and Exchange. Exploring relationships among places, people and things (pp. 49-68). Walnut Creek: Left Coast Press.

» Lema, C. (2004). Tebenquiche Chico en los siglos XVI y XVII. (Tesis de Licenciatura inédita), Universidad Nacional de Rosario, Argentina.

»Lema, V. (2008). ¿De qué hablamos cuando hablamos de domesticación vegetal en el NOA? Revisión de antiguas propuestas bajo nuevos abordajes teóricos. En S. Archila, M. Giovannetti y V. Lema (Eds.), Arqueobotánica y teoría arqueológica. Discusiones desde Suramérica (pp. 97-126). Bogotá: UNIANDES-CESO.

»Lema, V. (2013). Criar y ser criados por las plantas y sus espacios en los Andes septentrionales de Argentina. En A. Benedeti y J. Tomasi (Comp.), Espacialidades altoandinas. Avances de investigación desde el Noroeste argentino. Miradas hacia lo local, lo comunitario y lo doméstico (Tomo I) (pp. 301-338). Buenos Aires: Editorial de la Facultad de Filosofía y Letras, Universidad de Buenos Aires. 
» Llobera, M. (2012). Life on a pixel: Challenges in the development of digital methods within an "Interpretive" landscape archaeology framework. Journal of Archaeological Method and Theory, 19, 495-509

»López, G. (2003). Pastoreo y caza de camélidos en el Temprano de la Puna de Salta: Datos osteométricos del sitio Matancillas 2. Intersecciones en Antropología, 4, 17-27.

»López, G. (2006). Resultados del análisis arqueofaunístico de un basural, un recinto y un sector interrecinto del sitio Matancillas 2, Puna de Salta. Intersecciones en Antropología, 7, 207-216.

» Martínez, J. (2003). Ocupaciones humanas tempranas y tecnología de caza en la microrregión de Antofagasta de la Sierra (10000-700o AP). (Tesis Doctoral inédita), Universidad Nacional de Tucumán, Argentina.

» Maschner, H. (Ed.). (1996). New methods, old problems: geographic information systems in modern archaeological research. Illinois: Southern Illinois University.

» Mengoni Goñalons, G. y Yacobaccio, H. (2006). The domestication of South American camelids: A view from the South-Central Andes. En M. Zeder, D. Bradley, E. Emshwiller y B. Smith (Eds.), Documenting Domestication: New Genetic and Archaeological Paradigms (pp. 228-246). Berkeley: University of California Press.

» Moreno, E. (2005). Artefactos y prácticas. Análisis tecno-funcional de los materiales líticos de Tebenquiche Chico 1. (Tesis de Licenciatura inédita), Universidad Nacional de Catamarca, Argentina.

» Moreno, E. (2010). Arqueología de la caza de vicuñas en el área del Salar de Antofalla, Puna de Atacama. Una aproximación desde la arqueología del paisaje. (Tesis Doctoral inédita), Universidad Nacional de La Plata, Argentina.

» Moreno, E. (2011). Tecnología de caza en la quebrada de Antofalla. Revista del Museo de Antropología, 4, 17-32.

» Moreno, E. (2012). Propiciando el encuentro. La estructuración de los paisajes de cacería en el contexto andino. Intersecciones en Antropología, 13, 327-343.

" Moreno, E. (2016). Hunting technology and prepared landscapes in South-Central Andes. Lithic Technology, 41(4), 268-292.

» Moreno, E. y Chaparro, M. (2010). Valoraciones sociales y estéticas de las rocas. Circulación de obsidiana en el Noroeste Argentino. Trabajo presentado en el XVII Congreso Nacional de Arqueología Argentina. Mendoza, Argentina.

» Moreno, E. y Lema, C. (2012). El aprovechamiento de la vicuña en Tebenquiche Chico, Siglos XVI y XVII d.C. Aportes Científicos desde Humanidades, 9, 242-253.

" Moreno, E. y Quesada, M. (2012). Construcción de paisajes cinegéticos y relaciones sociales de larga duración en Antofalla. En P. Babot, M. Marschoff y F. Pazzarelli (Eds.), Las manos en la masa. Arqueologías y antropologías de la alimentación en Suramérica (pp. 583-604). Córdoba: Universidad Nacional de Córdoba y Universidad Nacional de Tucumán.

»Olivera, D. (1991). Tecnología y estrategias de adaptación en el formativo (agro-alfarero temprano) de la Puna Meridional Argentina. Un caso de estudio: Antofagasta de la Sierra (Pcia. de Catamarca, R. A.). (Tesis Doctoral inédita), Universidad Nacional de La Plata, Argentina.

» Olivera, D. (1997). La importancia del recurso Camelidae en la Puna de Atacama entre los 10000 y 500 años A.P. Estudios Atacameños, 14, 29-41. 
» Olivera, D., Escola, P., Elias, A., Tchilinguirian, P., Salminci, P., Perez, M., Grana, L., Grant, J., Vidal, A., Killian Galván, V. y Miranda, P. (2015). El formativo en la puna meridional. De la opción productiva a las sociedades agropastoriles plenas. En A. Korstanje, M. Lazzari, M. Basile, F. Bugliani, V. Lema, L. Pereyra Domingorena y M. Quesada (Eds.), Crónicas Materiales Precolombinas. Arqueología de los primeros poblados del Noroeste Argentino (pp. 663-691). Buenos Aires: Sociedad Argentina de Antropología.

» Pastor, S., Murrieta Flores, P. y García Sanjuán, L. (2013). Los SIG en la arqueología de habla hispana. Temas, técnicas y perspectivas. Comechingonia, 17(2), 9-29.

»Quesada, M. (2007). Paisajes Agrarios en el Área de Antofalla. Procesos de trabajo y escalas sociales de la producción agrícola. (Primer y segundo milenio d.C.). (Tesis Doctoral inédita), Universidad Nacional de La Plata, Argentina.

»Quesada, M. (2010). Agricultura Campesina en el Área de Antofalla. En A. Korstanje y M. Quesada (Eds.), Arqueología de la Agricultura. Casos de Estudio en la Región Andina Argentina (pp. 76-103). San Miguel de Tucumán: Editorial Magna.

»Quesada, M., Granizo, M. y Moreno, E. (2007). Estudio arqueológico del potencial agrícola en el área de Antofalla, Antofagasta de la Sierra, Catamarca. $1^{\circ}$ y $2^{\circ}$ Milenios d.C. Aportes científicos desde Humanidades, 7, 297-315.

» Ratto, N. (1994). Funcionalidad versus adscripción cultural: cabezales líticos de la margen norte del estrecho de Magallanes. En J. Lanata y L. Borrero (Eds.), Arqueología de Cazadores-recolectores. Límites, casos y aperturas (pp. 105-120). Buenos Aires: Ayllu.

»Ratto, N. (2003). Estrategias de caza y propiedades del registro arqueológico en la Puna de Chaschuil (Departamento de Tinogasta, Catamarca, Argentina). (Tesis Doctoral inédita), Universidad de Buenos Aires, Argentina.

»Rengifo, G. (1997). Diversidad y derecho de propiedad en los Andes. En J. Van Kessel y H. Larraín Barros (Eds.), Manos sabias para criar la vida. Tecnología andina (pp. 15-32). Quito: Abya-Yala - IECTA.

» Revuelta, C. (2005). Apropiación social y vicuñas. Análisis zooarqueológico de la unidad doméstica TC1. Oasis de Tebenquiche Chico-Primer milenio d.C. (Tesis de Licenciatura inédita), Universidad Nacional de Catamarca, Argentina.

»Shott, M. (1997). Stone and shafts redux: the metric discrimination of chipped-stone dart and arrow points. American Antiquity, 62(1), 86-101.

» Tomasi, J. (2011). La casa como una construcción múltiple y colectiva. Aproximación al espacio doméstico en Susques y Rinconada. En J. Tomasi y C. Rivet (Eds.), Puna y arquitectura. Las formas locales de la construcción (pp. 41-52). Buenos Aires: Centro de Documentación de Arquitectura Latinoamericana.

» Ucko, P. y Layton, R. (1999). The Archaeology and Anthropology of Landscape: Shaping your Landscape. Londres: Routledge.

»Van Kessel, J. y Condori Cruz, D. (1992). Criar la vida. Trabajo y tecnología en el mundo andino. Santiago: Vivarium.

»Vicent García, J. (1991). El neolítico. Transformaciones sociales y económicas. Boletín de Antropología Americana, 24, 31-61.

» Yacobaccio, H., Catá, M., Morales, M., Solá, P., Alonso, M., Rosenbusch, M., Vázquez, C., Samec, C., Oxman, B. y Cáceres, M. (2011). El uso de cuevas por pastores andinos: El caso de Cueva Quispe (Susques, Puna de Jujuy). En G. López y H. Muscio (Eds.), Arqueología de la Puna Argentina. Perspectivas actuales en el estudio de la diversidad y el cambio cultural (pp. 33-47). Oxford: BAR International Series 2296, Archaeopress. 
» Yacobaccio, H., Escola, P., Pereyra, F., Lazarri, M. y Glascock, M. (2004). Quest for ancient routes: obsidian sourcing research in Northwestern Argentina. Journal of Archaeological Science, 31, 193-204.

» Yacobaccio, H y Madero, C. (1992). Zooarqueología de Huachichocana III (Jujuy, Argentina). Arqueología, 2, 149-188.

» Yacobaccio, H., Madero, C., Malmierca, C. y Reigadas, M. (1997-1998). Caza, domesticación y pastoreo de camélidos en la Puna Argentina. Relaciones de la Sociedad Argentina de Antropología, XXII-XXIII, 389-418.

" Yacobaccio, H. y Vilá, B. (2002). Condiciones, mecanismos y consecuencias de la domesticación de los camélidos. Estudios Sociales del NOA, 5(5), 4-27. 